\title{
Interaction of mental and orthostatic stressors
}

\author{
Nandu Goswami ${ }^{\mathrm{a}, *}$, Helmut K. Lackner ${ }^{\mathrm{a}}$, Ilona Papousek ${ }^{\mathrm{c}}$, Daniela Jezova ${ }^{\mathrm{d}}$, \\ Jean-Pierre Montani ${ }^{\mathrm{e}}$, Helmut G. Hinghofer-Szalkay ${ }^{\mathrm{a}, \mathrm{b}}$ \\ a Institute of Physiology, Center of Physiological Medicine, Medical University of Graz, Harrachgasse 21/5, Graz, Austria \\ ${ }^{\mathrm{b}}$ Institute of Adaptive and Spaceflight Physiology, Wormgasse 9, Graz, Austria \\ c Department of Psychology, Karl Franzens University, Graz, Austria \\ d Institute of Experimental Endocrinology, Slovak Academy of Sciences, Bratislava, Slovakia \\ e Department of Medicine/Physiology, University of Fribourg, Switzerland
}

\begin{abstract}
We assessed hemodynamic responses induced by orthostatic and mental stressors, using passive head up tilt (HUT) and mental arithmetic (MA), respectively. The 15 healthy males underwent three protocols: (1) HUT alone, (2) MA in supine position and (3) MA+HUT, with sessions randomized and $\geq 2$ weeks apart. In relation to baseline, HUT increased heart rate (HR) $(+20.4 \pm 7.1 \mathrm{bpm} ; p<0.001)$, mean blood pressure (MBP) $(+4.7 \pm 11.3 \mathrm{mmHg} ; p<0.05)$, diastolic blood pressure (DBP) $(+6.1 \pm 11.6 \mathrm{mmHg}$; $p<0.05)$ and total peripheral resistance (TPR) $\left(+155 \pm 232\right.$ dyne $\left.^{*} \mathrm{~s} / \mathrm{cm}^{5} ; p<0.001\right)$ but decreased stroke volume (SV) $(-33.1 \pm 13.4 \mathrm{ml} ; p<0.001)$ and cardiac output (CO) $(-0.6 \pm 1.01 / \mathrm{min} ; p<0.01)$. MA increased HR $(+8.0 \pm 6.0 \mathrm{bpm} ; p<0.001)$, systolic blood pressure (SBP) $\quad(+9.0 \pm 7.7 \mathrm{mmHg} ; \quad p<0.001), \quad \mathrm{MBP} \quad(+10.0 \pm 6.5 \mathrm{mmHg}$; $p<0.001)$, DBP $(+9.5 \pm 7.2 \mathrm{mmHg} ; p<0.001)$ and $\mathrm{CO}(+0.6 \pm 0.8 \mathrm{l} / \mathrm{min} ; p<0.01)$. $\mathrm{MA}+\mathrm{HUT}$ increased HR $(+28.8 \pm 8.4 \mathrm{bpm} ; \quad p<0.001)$, SBP $\quad(+4.6 \pm 14.3 \mathrm{mmHg}$; $p<0.05), \operatorname{MBP}(+11.2 \pm 11.6 \mathrm{mmHg} ; p<0.001)$, DBP $(+13.5 \pm 10.1 \mathrm{mmHg} ; p<0.001)$ and TPR $\left(+160 \pm 199\right.$ dyne*s $\left./ \mathrm{cm}^{5} ; p<0.001\right)$ but SV $(-34.5 \pm 14.6 \mathrm{ml} ; p<0.001)$ decreased. Mental challenge during orthostatic challenge elicited greater increases in heart rate, despite similar reductions in stroke volume such as those during orthostatic stress alone. Overall, cardiac output decreases were less with combinations of mental and orthostatic challenges in comparison to orthostasis alone. This would suggest that carefully chosen mental stressors might affect orthostatic responses of people on standing up. Therefore, additional mental loading could be a useful countermeasure to alleviate the orthostatic responses of persons, particularly in those with histories of dizziness on standing up or on return to earth from the spaceflight environment of microgravity.
\end{abstract}

\section{Introduction}

Mental stress induces hemodynamic, autonomic and hormonal responses [1]. Mental challenge activates the sympathetic system, increases heart rate, cardiac output

\footnotetext{
* Corresponding author. Tel.: +433163804278.

E-mail address: Nandu.goswami@meduni-graz.at (N. Goswami).
}

and blood pressure, and plays an important part in controlling resistance vessels [2]. Mental arithmetic (MA) is used regularly to provide mental challenge.

We have recently reported that orthostatic responses are not different when head up tilt (HUT) is preceded by mental challenge [3]. However, what is not known is whether simultaneous application of mental challenge in orthostatically challenged subjects would have an effect on cardiovascular responses. This is important as in daily 
life, mental and physical stresses combine. While there are similarities in responses induced by these stressors, important differences do exist [4]. For example, mental stress, by increasing central command, affects arterial baroreceptors loading [5]. Orthostatic stress induced central hypovolemia, on the other hand, leads to cardiopulmonary baroreceptor unloading. Based on these observations, we hypothesized that combination of mental and orthostatic challenges may alter physiological responses. The additional effects of MA on subjects in HUT would provide novel insights into the modulation of orthostatic cardiovascular responses by mental challenge. This could be a useful countermeasure to alleviate the orthostatic responses of persons, particularly in those with histories of dizziness upon standing up or on return to earth from the spaceflight environment of microgravity.

We assessed hemodynamic responses induced by orthostatic and mental stressors, using HUT and MA, respectively. HUT alone data are from our previous study [3]. The same participants underwent MA alone and MA+HUT protocols.

\section{Materials and methods}

\subsection{Participants}

Because gender and age may affect orthostatic and stress responses (reviewed in [6]), we focused on young healthy men whose physical characteristics were homogeneous. The study criteria were met by 15 Caucasian males of age $27 \pm 5 \mathrm{yrs}$, weight $74 \pm 8 \mathrm{~kg}$, height $179 \pm$ $5 \mathrm{~cm}$, and a heart rate of $60 \pm 6 \mathrm{bpm}$ during supine rest $($ mean $\pm S D)$.

Every day, between 9-11 AM and 11 AM-1 PM), two subjects did any one of the three protocols: (1) HUT alone, (2) MA in supine position and (b) MA+HUT, with sessions randomized and $\geq 2$ weeks apart. Stress exposure was for $10 \mathrm{~min}$ in all the protocols. Subjects were familiarized with the test protocols and gave written informed consent to participate in the study. The study was approved by the Graz University Ethics Board and the study performed in accordance with the 1989 WMA Declaration of Helsinki.

\subsection{Study design}

We used a symmetric, crossover design with an online randomizer allocating the subjects to each protocol. The subjects served as their own control. We asked participants to abstain from coffee and other stimulants for 2 days before the test sessions.

At the beginning of the selection process, the subjects were informed about the three protocols; however, they were not told in advance of the order of the protocols. During their first visit, the subjects were familiarized with the laboratory, personnel and equipment. They received standardized verbal instructions about the protocol, tasks, and computer administered mental arithmetic. Participants were told that they should solve the tasks as accurately and as fast as possible and that the answers were recorded. A timer applied additional pressure. Halfway through the mental arithmetic, subjects were asked to answer more correctly, irrespective of their correct answers. These procedures were designed to help reduce adaptation to the stress condition. No external feedback regarding performance during the MA was provided during the study.

The tests were carried out in a semi-dark and quiet room, maintained at $24^{\circ} \mathrm{C}$ and humidity at 55\%, using an electronically driven tilt table [7].

\subsubsection{Orthostatic challenge}

The orthostatic challenge was provided by HUT. A 30 min supine rest preceded each experiment. At minute zero, the tilt table was brought to $70^{\circ}$ head-up position and after $10 \mathrm{~min}$ the table was returned to supine position. During the test the subjects were supported by an adjustable footrest and were instructed to avoid undue movements of the lower limbs and to breathe normally.

Since the aim of the experiment was to induce orthostatic stress without inducing syncope, criteria of termination included any of the following [8]: (a) presyncope in hemodynamically defined terms-blood pressure falling below systolic $80 \mathrm{mmHg}$, or that it dropped rapidly (systolic by $\geq 20 \mathrm{mmHg} / \mathrm{min}$, diastolic by $\geq 10 \mathrm{mmHg}$ / min), or heart rate dropped by $\geq 15 \mathrm{bpm}$; (b) Lightheadedness, dizziness, visual disturbances, nausea, stomach awareness, clammy skin, excessive sweating, or skin pallor. However, all the subjects went through all the protocols with no problems.

\subsubsection{Mental challenge}

Provided by MA. Subjects subtracted continuously the numbers 6 or 7, randomly, from a 2 or 3 digit number while lying supine [1]. A new number was provided every $5 \mathrm{~s}$ to subtract from on a computer screen fixed at the eyes of the subjects.

\subsubsection{Combined orthostatic and mental challenge $(M A+H U T)$}

MA was started immediately upon assumption of the upright posture (HUT), and was ended when subject returned to supine position.

Fig. 1 depicts the MA+HUT protocol used.

\subsection{Self reported measures}

Emotional status was assessed on arrival at the laboratory using the State-Trait anxiety inventory [9] and the General Depression scale [10].

Performance (mistakes made) on the task was assessed and ratings of perception of stress (PSS), shortly before commencing the stresses and retrospectively at the end of mental/orthostatic stress, was done using a 5 point scale (1: not stressful; 5: very, very stressful) [1].

\subsection{Recording physiological stress responses}

Baseline data were collected for $30 \mathrm{~min}$ with the subjects in supine position. During baseline, the subjects were 


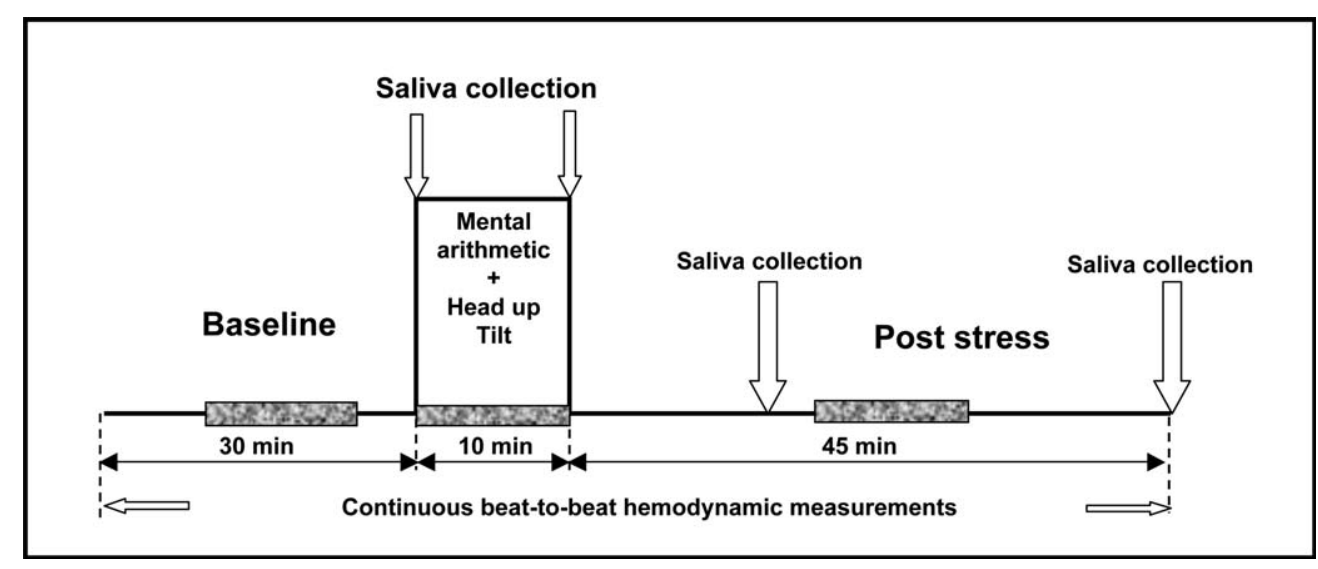

Fig. 1. MA+HUT protocol. MA: mental arithmetic; HUT: Head up tilt. Saliva collection was done at end of 30 min baseline (sample 1 ), b) after finishing MA and/or MA+HUT (sample 2) and at 15 (sample 3) and $45 \mathrm{~min}$ (sample 4) after termination of challenges. Rectangular hatched areas represent durations (10 min each) during which data were analyzed.

requested to relax without falling asleep. After the stress period, physiological data were recorded for $45 \mathrm{~min}$.

\subsubsection{Hemodynamic monitoring}

Hemodynamic monitoring included blood pressure (upper arm oscillometry and finger plethysmography), heart rate (3-lead ECG) and thoracic impedance measurements using a Task Force Monitor ${ }^{\circledR}$ (TFM, CNSystems, Graz, Austria). For the variables related to impedance cardiography beat to beat values computed by the TFM ${ }^{\circledR}$ were used. Thoracic impedance $\mathrm{ZO}(\mathrm{t})$ and impedance variation $\mathrm{dZ}(\mathrm{t}) / \mathrm{dt}$ were used to calculate beat-to-beat stroke volume based on an improved Kubicek approach and cardiac output. Total peripheral resistance (TPR) was calculated as $80 \times$ (mean arterial blood pressure-central venous pressure)/cardiac output [11]. TFM ${ }^{\circledR}$ ECG/impedance electrodes were positioned at the neck and thoracic regions, the latter at the midclavicular line at the xiphoid process level [12].

\subsubsection{Saliva collection}

Salivary cortisol and alpha amylase were measured (detailed in [3]). Saliva collection was after $30 \mathrm{~min}$ of baseline, completion of HUT, MA, or MA+HUT and at 15and $45 \mathrm{~min}$ post stress (Fig. 1), using the Salivette ${ }^{\circledR}$ system. Samples were frozen to $-30^{\circ}$ and measured using standard reagent kits not later than one month after sampling.

\subsection{Sample size and data analysis}

Using typical cardiovascular changes during orthostatic loading from previous studies [3,13], error probability $(\alpha)$ of 0.05 , power $(1-\beta)$ of 0.80 and considering an average effect size (d) of 0.5 , we estimated the number of subjects required to be 15 .

All calculations were made with Matlab R2007 (The MathWorks Inc.) and SPSS version 16. Each protocol lasted $85 \mathrm{~min}$. Data were analyzed in $10 \mathrm{~min}$ frames representing baseline, stress application and post stress period (15-25, 35-45 and 60-70 min, respectively) (Fig. 1). Data are expressed as mean $+\mathrm{SD}$.

To evaluate the differences induced by mental and orthostatic stressors, $3 \times 3$ analyses of variance (ANOVAs) were conducted, with phase/condition (baseline, stress, post stress) and protocol/type of stress (HUT only, HUT combined with mental challenge, MA in supine position) as within-subjects factors, and the cardiovascular measures as the dependent variables, followed by post hoc tests (Tukey's Honestly Significant Difference, HSD). Separate analyses were conducted for selected hemodynamic variables (HR, SV, CO), blood pressure variables (SBP, DBP and MBP) and vascular resistance (TPR), respectively. Two 4 (phase: baseline, HUT, 15 min post stress, $45 \mathrm{~min}$ post stress; within-subject factor) $\times 3$ (protocol; within-subject factor) ANOVAs were performed to analyze effects on alpha-amylase and cortisol responses, respectively.

ANOVA was used for comparing stress application minus baseline for MA+HUT and the value for MAHUT ((stress application minus baseline for HUT)+(stress application minus baseline for MA)). Additionally, Pearson product-moment correlation coefficient was used to analyze the correlation between these two variables.

Paired two-sided Wilcoxon signed rank test was used for comparing the mistakes made during $\mathrm{MA}$ in the protocols MA+HUT and MA. We used the non-parametric Friedman test to analyze perception of stress (PSS) between the baselines of HUT, MA+HUT and MA. Similarly, differences in PSS between during- and beginning of HUT, MA+HUT and MA were compared.

\section{Results}

Significant main effects of phase on all cardiovascular variables (heart rate, stroke volume and cardiac output: $F(6,9)=38.4, p<0.001$; systolic, diastolic, and mean BP: $F(6,9)=18.3, p<0.001$; and total peripheral resistance: $F(2,13)=7.2, p<0.01$ were seen. Subsequently performed 
a

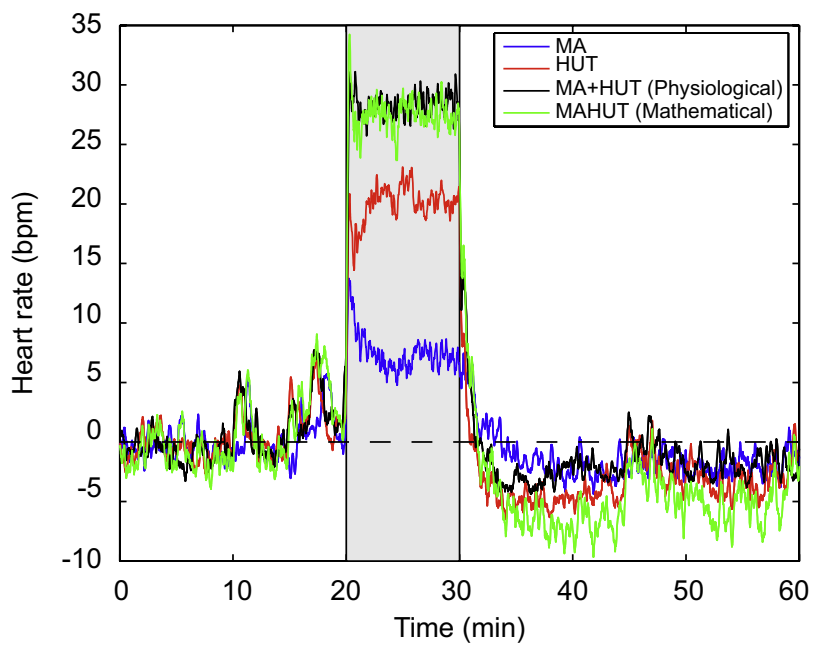

C

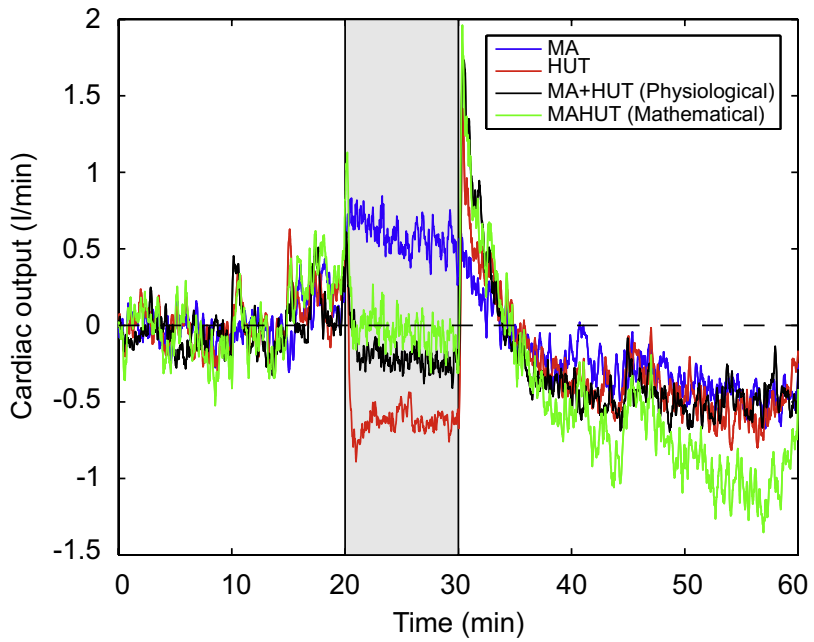

b

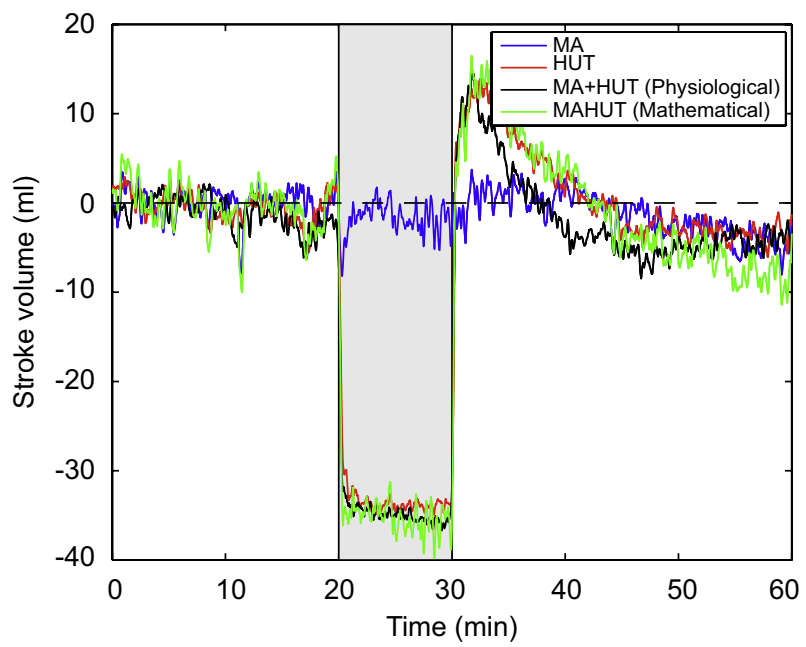

d

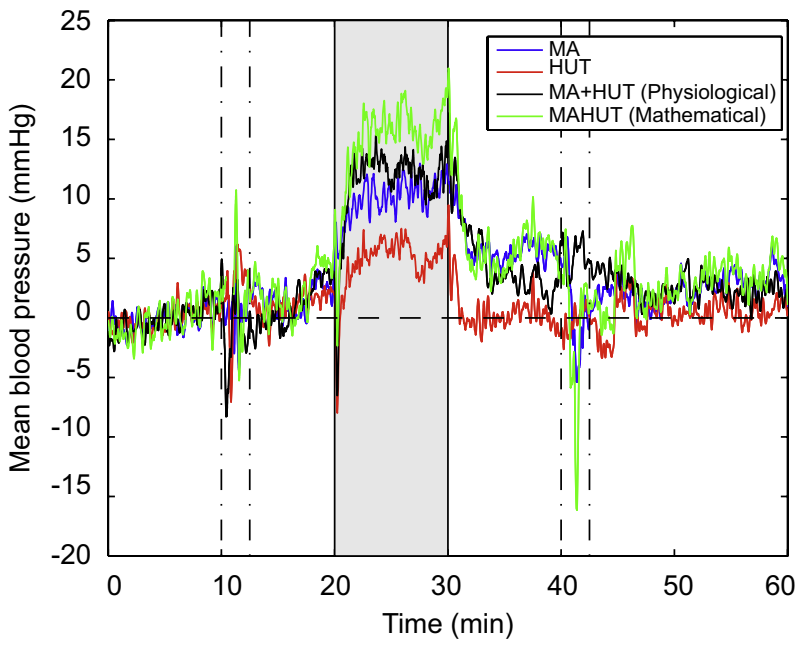

Fig. 2. Relative changes (in comparison to the baseline) of the means of all subjects for selected hemodynamic variables across each protocol: (A) heart rate; (B) stroke volume; (C) cardiac output and (D) mean blood pressure. In the area between the dashed lines, in baseline and post stress periods, blood pressure was recalibrated by the Task force monitor. Shaded area refers to the stress application.

univariate $F$-tests showed that the significances were seen for all cardiovascular variables.

HUT alone increased heart rate (HR) $(+20.4 \pm 7.1$ bpm; $p<0.001)$, mean blood pressure (MBP) $(+4.7 \pm$ $11.3 \mathrm{mmHg} ; p<0.05)$, diastolic blood pressure (DBP) $(+6.1 \pm 11.6 \mathrm{mmHg} ; p<0.05)$ and total peripheral resistance (TPR) $\left(+155 \pm 232\right.$ dyne $\left.^{*} \mathrm{~s} / \mathrm{cm}^{5} ; p<0.001\right)$ but decreased stroke volume (SV) $(-33.1 \pm 13.4 \mathrm{ml} ; p<0.001)$ and cardiac output $(\mathrm{CO})(-0.6 \pm 1.0 \mathrm{l} / \mathrm{min} ; p<0.01)$.

MA in supine position increased HR $(+8.0 \pm 6.0 \mathrm{bpm}$; $p<0.001)$, systolic blood pressure (SBP) $(+9.0 \pm 7.7 \mathrm{mmHg}$; $p<0.001), \quad$ MBP $\quad(+10.0 \pm 6.5 \mathrm{mmHg} ; \quad p<0.001), \quad$ DBP $(+9.5 \pm 7.2 \mathrm{mmHg} ; p<0.001)$ and $\mathrm{CO}(+0.6 \pm 0.8 \mathrm{l} / \mathrm{min}$; $p<0.01$ ).

$\mathrm{MA}+\mathrm{HUT}$ increased HR $(+28.8 \pm 8.4 \mathrm{bpm} ; p<0.001)$, SBP $(+4.6 \pm 14.3 \mathrm{mmHg} ; \quad p<0.05), \quad$ MBP $(+11.2 \pm 11.6$ mmHg; $p<0.001)$, DBP $(+13.5 \pm 10.1 \mathrm{mmHg} ; p<0.001)$ and TPR $\left(+160 \pm 199\right.$ dyne* $\left.^{*} / \mathrm{cm}^{5} ; \quad p<0.001\right)$ but SV $(-34.5 \pm 14.6 \mathrm{ml} ; p<0.001)$ decreased.

Significant main effects of protocol and interaction phase $\mathrm{x}$ protocol were seen in heart rate, stroke volume and cardiac output $(F(6,9)=10.7, \quad p<0.01$ and $F(12,3)=$ $11.6, p<0.05$, respectively). Subsequently performed univariate $F$-tests showed that significances for heart rate, stroke volume and cardiac output.

The post hoc tests for the interaction of phase $x$ protocol showed different time courses of heart rate between the three protocols. However, the decrease of the cardiac output relative to baseline was lower in MA+HUT compared to HUT alone $(p<0.05)$.

For some hemodynamic variables such as HR (Fig. 2a), SV (Fig. 2b) and CO (Fig. 2c), the responses induced by simultaneous application of the individual stressors (MA+HUT) were consistent with the mathematical sum 
Table 1

Means and standard deviations of some hemodynamic variables.

\begin{tabular}{|c|c|c|c|c|c|c|}
\hline & Baseline & Stress & Post stress & Stress-baseline & ANOVA & Correlation \\
\hline \multicolumn{7}{|c|}{ Heart rate (bpm) } \\
\hline HUT & $60.3 \pm 7.9$ & $80.8 \pm 10.9$ & $57.9 \pm 7.0$ & $20.4 \pm 7.1$ & & \\
\hline MA & $64.7 \pm 8.9$ & $72.7 \pm 11.6$ & $63.3 \pm 7.1$ & $8.0 \pm 6.0$ & & \\
\hline $\mathrm{MA}+\mathrm{HUT}$ & $62.1 \pm 4.8$ & $90.9 \pm 8.1$ & $61.1 \pm 7.4$ & $28.8 \pm 8.4$ & & \\
\hline MAHUT & & & & $28.4 \pm 10.6$ & $\mathbf{F}=0.024 \mathrm{~ns}$ & $\boldsymbol{R}=0.503 \boldsymbol{p}<0.10$ \\
\hline \multicolumn{7}{|c|}{ Stroke volume (ml) } \\
\hline HUT & $105.3 \pm 16.2$ & $72.1 \pm 8.7$ & $102.1 \pm 16.5$ & $-33.1 \pm 13.4$ & & \\
\hline MA & $101.6 \pm 16.0$ & $99.6 \pm 16.5$ & $99.2 \pm 15.8$ & $-2.0 \pm 7.9$ & & \\
\hline $\mathrm{MA}+\mathrm{HUT}$ & $104.3 \pm 14.4$ & $69.7 \pm 6.9$ & $98.9 \pm 16.1$ & $-34.5 \pm 14.6$ & & \\
\hline MAHUT & & & & $-35.1 \pm 15.9$ & $\mathbf{F}=0.049 \mathrm{~ns}$ & $\boldsymbol{R}=0.786 \boldsymbol{p}<0.01$ \\
\hline \multicolumn{7}{|c|}{ Cardiac output (1/min) } \\
\hline HUT & $6.3 \pm 1.3$ & $5.8 \pm 0.8$ & $5.9 \pm 1.2$ & $-0.6 \pm 1.0$ & & \\
\hline MA & $6.6 \pm 1.5$ & $7.2 \pm 1.6$ & $6.3 \pm 1.3$ & $0.6 \pm 0.8$ & & \\
\hline $\mathrm{MA}+\mathrm{HUT}$ & $6.5 \pm 1.0$ & $6.3 \pm 0.8$ & $6.0 \pm 1.1$ & $-0.2 \pm 1.0$ & & \\
\hline MAHUT & & & & $0.1 \pm 1.3$ & $\mathbf{F}=0.515 \mathrm{~ns}$ & $\boldsymbol{R}=0.544 \boldsymbol{p}<0.05$ \\
\hline \multicolumn{7}{|c|}{ Systolic blood pressure (mmHg) } \\
\hline HUT & $118.7 \pm 8.7$ & $117.2 \pm 13.8$ & $116.2 \pm 11.6$ & $-1.5 \pm 11.6$ & & \\
\hline MA & $118.3 \pm 12.3$ & $127.3 \pm 13.8$ & $119.4 \pm 11.8$ & $9.0 \pm 7.7$ & & \\
\hline $\mathrm{MA}+\mathrm{HUT}$ & $122.6 \pm 10.4$ & $127.2 \pm 17.2$ & $120.7 \pm 9.9$ & $4.6 \pm 14.3$ & & \\
\hline MAHUT & & & & $7.6 \pm 14.7$ & $\mathbf{F}=0.295 \mathrm{~ns}$ & $\boldsymbol{R}=-0.05 \mathrm{~ns}$ \\
\hline \multicolumn{7}{|c|}{ Mean blood pressure (mmHg) } \\
\hline HUT & $86.7 \pm 8.5$ & $91.2 \pm 14.1$ & $87.0 \pm 10.0$ & $4.7 \pm 11.3$ & & \\
\hline MA & $87.1 \pm 11.3$ & $97.0 \pm 11.8$ & $88.9 \pm 10.4$ & $10.0 \pm 6.5$ & & \\
\hline $\mathrm{MA}+\mathrm{HUT}$ & $89.2 \pm 10.2$ & $100.4 \pm 13.2$ & $91.6 \pm 10.5$ & $11.2 \pm 11.6$ & & \\
\hline MAHUT & & & & $14.7 \pm 15.5$ & $\mathbf{F}=0.203 \mathrm{~ns}$ & $\boldsymbol{R}=0.141 \mathrm{~ns}$ \\
\hline \multicolumn{7}{|c|}{ Diastolic blood pressure (mmHg) } \\
\hline HUT & $71.5 \pm 8.6$ & $77.5 \pm 12.8$ & $72.8 \pm 9.1$ & $6.1 \pm 11.6$ & & \\
\hline MA & $72.3 \pm 11.5$ & $81.7 \pm 11.3$ & $75.5 \pm 10.2$ & $9.5 \pm 7.2$ & & \\
\hline $\mathrm{MA}+\mathrm{HUT}$ & $73.5 \pm 10.2$ & $86.9 \pm 11.3$ & $77.1 \pm 10.1$ & $13.4 \pm 10.1$ & & \\
\hline MAHUT & & & & $15.5 \pm 17.0$ & $\mathbf{F}=0.059 \mathrm{~ns}$ & $\boldsymbol{R}=0.197 \mathrm{~ns}$ \\
\hline \multicolumn{7}{|c|}{ Total peripheral resistance (dyne* $/ \mathrm{cm}^{5}$ ) } \\
\hline HUT & $1110 \pm 268$ & $1265 \pm 299$ & $1193 \pm 271$ & $155 \pm 232$ & & \\
\hline MA & $1087 \pm 322$ & $1115 \pm 342$ & $1155 \pm 296$ & $27 \pm 134$ & & \\
\hline $\mathrm{MA}+\mathrm{HUT}$ & $1107 \pm 265$ & $1268 \pm 251$ & $1232 \pm 290$ & $160 \pm 199$ & & \\
\hline MAHUT & & & & $182 \pm 302$ & $\mathbf{F}=0.073 \mathrm{~ns}$ & $\boldsymbol{R}=0.298 \mathrm{~ns}$ \\
\hline
\end{tabular}

Bold values represent the mathematical sum of the responses (MA+HUT): (stress application minus baseline for HUT)+(stress application minus baseline for MA).

of the responses induced by single applications MA and HUT (that is, MA+HUT=MAHUT) (Table 1, Fig. 2). Mean arterial pressure did not, however, show this (Fig. 2d).

Fig. 3 is a cubical representation of the relationship of orthostatic and mental stressors, when applied singly or in combination. In 3A: red line refers to the HUT while blue line is the MA. In 3B: black line refers to the MA+HUT (physiological effects) while green line is the MAHUT (mathematical sum). In both these cubes, the shadowed area and hatch marks at the bottom of the figure panel represent stroke volume and heart rate, from which cardiac output can be calculated. When cardiac output is seen in relation to the other variable in the cube (total peripheral resistance) the mean arterial pressure can be obtained, according to the relationship: $\mathrm{MAP} \sim \mathrm{TPR} \times \mathrm{SV} \times \mathrm{HR}$.

Analyses of salivary responses showed no significant effects for alpha amylase and cortisol.

Perception of stress (PSS) increased in response to both mental and orthostatic challenges. Stress perception of the three protocols, however, was not different. The self reported variables and errors in calculation did not differ across the three stress conditions.

\section{Discussion}

The beneficial effect of mental arithmetic during head up tilt (MA+HUT) included a greater increase in heart rate than with mental- or orthostatic challenge alone. Theses findings are different to our previous observation regarding orthostatic stress preceded by mental challenge [3]: no differences in orthostatic responses were observed when mental challenge preceded orthostatic stress. To obtain beneficial effects of mental challenge on orthostatic responses, the timing of the mental loading appears to be important.

Orthostatic and mental stressors induce different physiological responses [4]. Indeed, the mechanisms of cardiovascular regulation have been reported to be different in the two (orthostatic and mental) forms of stress: cardiopulmonary baroreceptor unloading due to central hypovolemia occurs with orthostatic stress while an increase in central 


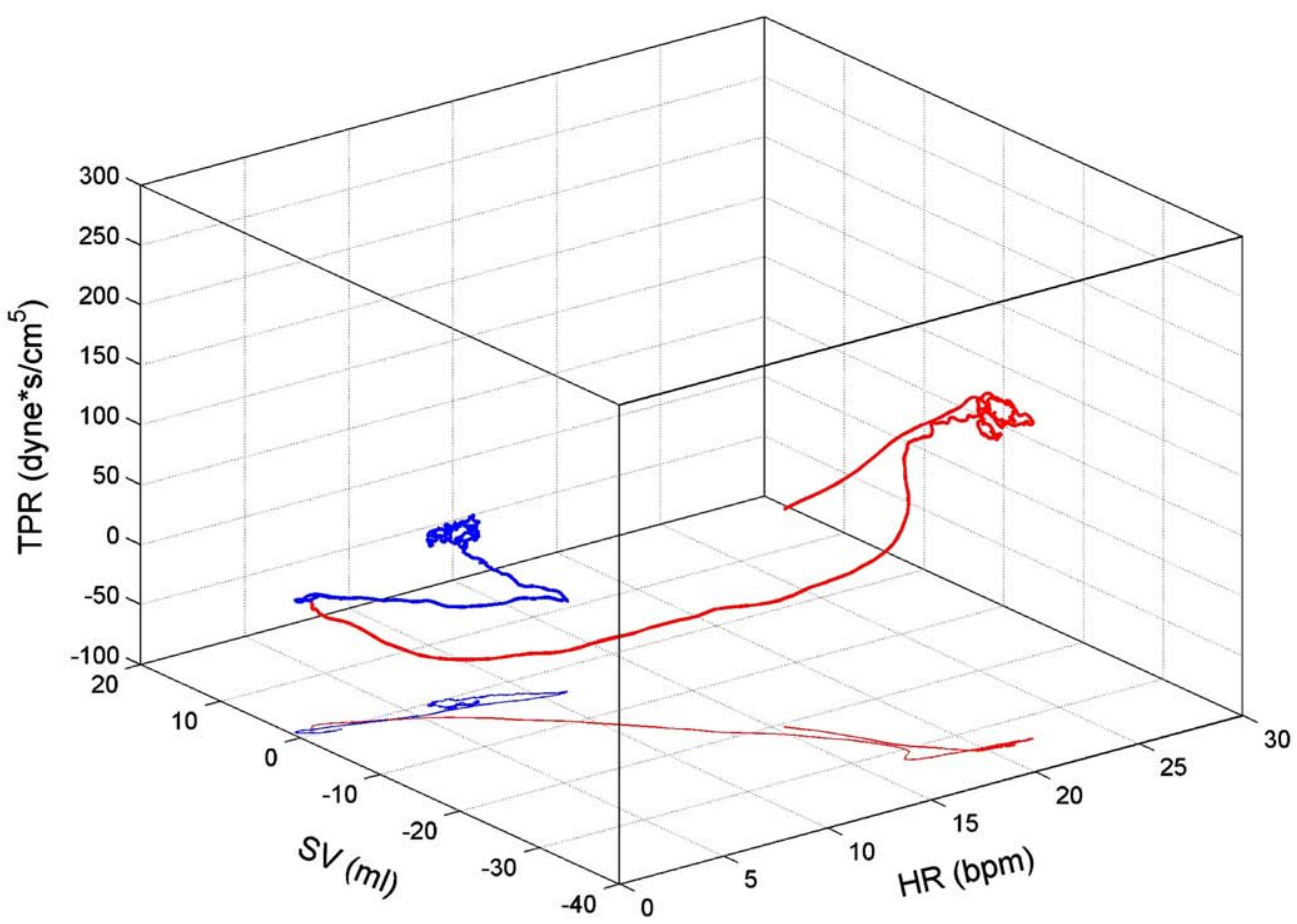

b

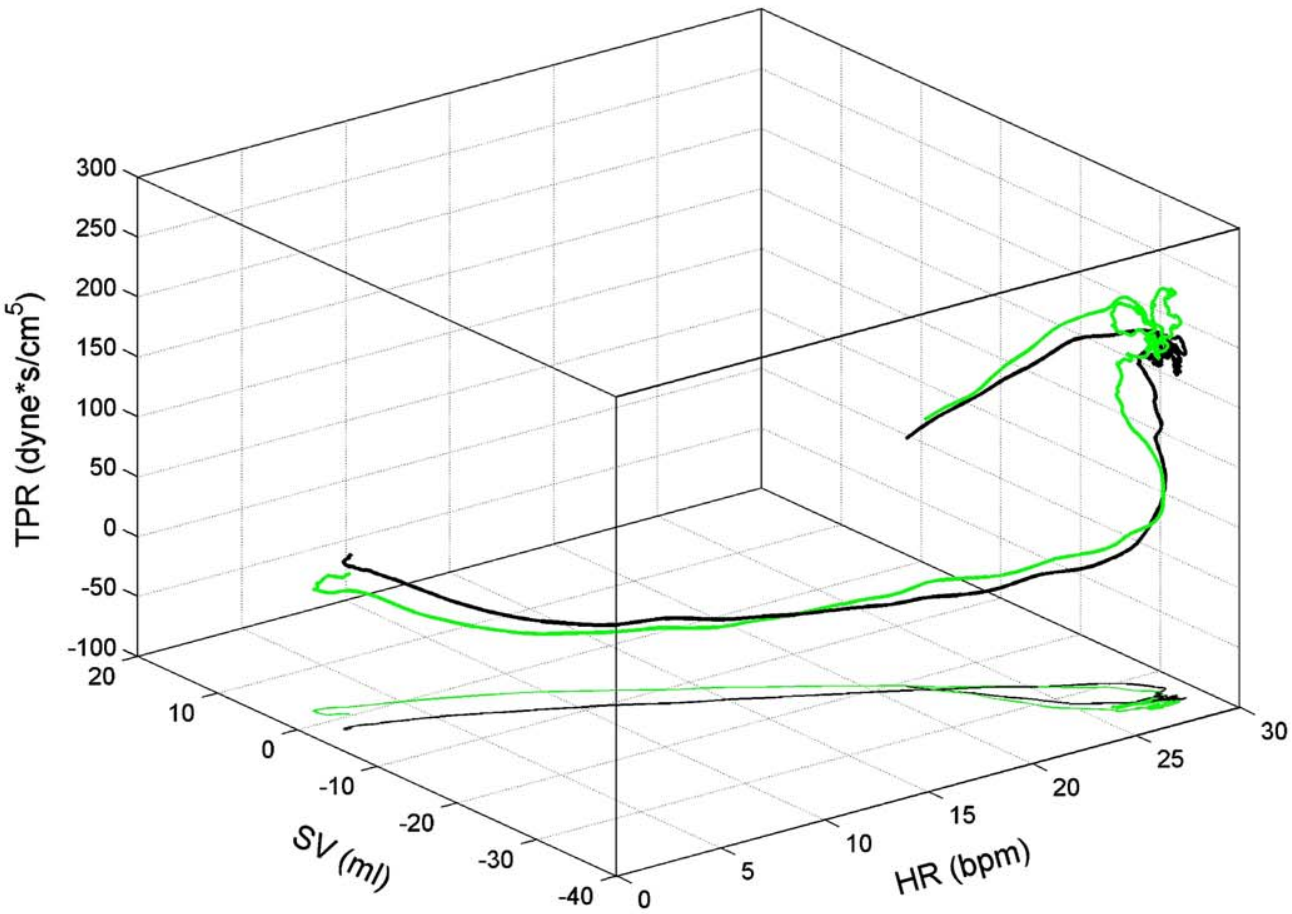

Fig. 3. Relationship of the stressors for selected $5 \mathrm{~min}$ (last $30 \mathrm{~s}$ of baseline and first $270 \mathrm{~s}$ of different stress application). Different relative responses (in other words the physiological behavior of responses) induced by the two stressors (applied singly) (Fig. 3A) (no correlation) or when they are combined (mathematically and physiologically) (3B) (high correlation). In 3A: red line refers to the HUT while blue line is the MA. In 3B: black line refers to the MA+HUT (physiological effects) while green line is the MAHUT (mathematical sum). In both these cubes, commencement of the responses is shown on the left hand side and the shadowed area and hatch marks at the bottom of the figure panel represent stroke volume and heart rate, from which cardiac output can be calculated. When cardiac output is seen in relation to the other variable in the cube (total peripheral resistance) the mean arterial pressure can be obtained, according to the relationship: MAP $\sim \mathrm{TPR} \times \mathrm{SV} \times \mathrm{HR}$. (For interpretation of the references to color in this figure legend, the reader is referred to the web version of this article.) 
command and arterial baroreceptor loading is noticed under mental stress [5]. It is plausible that during a combination of the two, the observed increases in heart rate are attributed to an increase in arterial baroreceptor unloading. Overall, the cardiac output (representative of global tissue perfusion in healthy populations) decreases were less with MA associated with HUT in comparison to HUT alone. This would suggest that carefully chosen mental stressors might affect the orthostatic responses of people on standing up. In addition, present results provide additional support to our previous suggestion [14] that physiological alterations observed in models combining mental stressors and changes in body posture, such as in public speaking, must be interpreted with caution.

Furthermore, for some hemodynamic variables such as heart rate, stroke volume and cardiac output the responses induced by simultaneous application of the individual stressors (MA+HUT) were consistent with the mathematical sum of the responses induced by single applications of mental (MA) and orthostatic challenge (HUT) (that is, MA+HUT = MAHUT), thereby validating the superposition principle. Mathematically speaking, the superposition principle states that the net response caused by two or more stimuli applied simultaneously is the sum of the responses caused by each individual stimulus applied alone [15]. For example, in a linear system represented as $y=f(x)$, the superposition principle is valid when:

$f\left(x_{1}+x_{2}\right)=f\left(x_{1}\right)+f\left(x_{2}\right)$

As physiological systems, when studied in vivo, are very complex and operate in non-linear and dynamic fashion, our results point to a novel observation. This implies that in a certain range of physiological states some hemodynamic variables, particularly the heart rate, stroke volume and the cardiac output respond linearly to stress application. Heart rate, stroke volume and cardiac output showed closer relationship to each other. However, mean blood pressure did not show any relationship, thereby implying that, at least for blood pressure changes, it is not possible to extrapolate from mathematical models to physiological systems. Observed response patterns to moderate physical and mental stressors used need not necessarily apply to more stressful stimuli or different stimuli combinations.

The additive nature of the physiological and psychological stressors on cardiovascular responses may not only be due to effects at the peripheral level but also at central (nervous system) level. This is particularly so, as the activation of cardiovascular control centres in the brainstem by signals from higher brain centres has been reported [16]. Physical and mental stressors may, however, exert their central effects differently. For example, metabolic demands of working muscles during physical activity such as standing up or exercise send ascending signals to the brain (bottomup) while mental arithmetic, requiring intense effort in working memory, and operating without regard to physiological demands, causes a top-down activation $[16,17]$.

In conclusion, mental challenge enhanced orthostatic cardiovascular responses in persons subjected to orthostatic stress. Additionally, we observed that there was an additive effect in selected hemodynamic responses during moderate stress induced by orthostatic and mental stressors. These results imply that mental challenge might improve blood pressure response in subjects upon standing up. We propose that mental arithmetic should be used as a tool to improve orthostatic tolerance. Indeed, pilot studies in our laboratory have shown that mental challenge improves orthostatic tolerance. Mental challenge might be a useful countermeasure particularly in those with histories of dizziness upon standing up or on return to earth from the spaceflight environment of microgravity.

Limitations

We could not discriminate effects of mental arithmetic from the known effects of just talking on blood pressure.

\section{Acknowledgements}

We wish to thank Dr Andreas Rössler, Dr Erik Grasser and Mr. Andreas Jantscher, all of Medical University of Graz, Austria for their excellent technical assistance during the experiments. We wish to also thank the participants for their time and patience.

\section{Funding}

This study was funded by the Austrian Research Promotion Agency (FFG project 817086 'Orthocap').

\section{Disclosures}

None.

\section{References}

[1] J.R. Carter, N.T. Kupiers, C.A. Ray, Neurovascular responses to mental stress, J Physio-London. 564 (2005) 321-327.

[2] D. Jezova, A. Makatsori, R. Duncko, F. Moncek, M. Jakubek, High trait anxiety in healthy subjects is associated with low neuroendocrine activity during psychosocial stress, Prog. Neuropsychopharmacol. Biol. Psychiatry 28 (2004) 1331-1336.

[3] N. Goswami, H.K. Lackner, I. Papousek, J.P. Montani, D. Jezova, H Hinghofer-Szalkay, Does mental arithmetic before head up tilt have an effect on the orthostatic cardiovascular and hormonal responses, Acta Astronautic., 2009, doi:10.1016/j.actaastro.2009.09.033.

[4] A. Kamiya, S. Iwase, D. Michikami, Q. Fu, T. Mano, Head-down bed rest alters sympathetic and cardiovascular responses to mental stress, Am. J. Physiol-Regulat. Integ. Comp. Physiol. 279 (2000) R440-R447.

[5] C.A. Sweene, M. Bootsma, H.H. Van Bolhuis, Different autonomic responses to orthostatic and to mental stress in young normals, Homeostasis 36 (1995) 287-292.

[6] N. Goswami, J.A. Loeppky, H. Hinghofer-Szalkay, Lbnp: past protocols and technical considerations for experimental design, Aviat. Space Environ. Med. 79 (2008) 459-471.

[7] N. Goswami, H.K. Lackner, E.K. Grasser, H.G. Hinghofer-Szalkay, Individual stability of orthostatic tolerance response, Acta Physiol. Hung. 96 (2009) 157-166.

[8] E.K. Grasser, N. Goswami, A. Rossler, K. Vreckoc, H. HinghoferSzalkay, Hemodynamic and neurohormonal responses to extreme orthostatic stress in physically fit young adults, Acta Astronautic. 64 (2009) 688-696.

[9] L. Laux, P. Glanzmann, P. Schaffner, C.D. Spielberger, Das StateTrait-Angstinventar, Beltz, Weinheim, 1981.

[10] M. Hautzinger, M. Bailer, Allgemeine Depressions Skala, Beltz, Weinheim, 1993.

[11] G. Gratze, J. Fortin, A. Holler, K. Grasenick, G. Pfurtscheller, P. Wach, J. Schonegger, P. Kotanko, F. Skrabal, A software package for 
non-invasive, real-time beat-to-beat monitoring of stroke volume, blood pressure, total peripheral resistance and for assessment of autonomic function, Comp. Biol. Med. 28 (1998) 121-142.

[12] J. Fortin, W. Habenbacher, A. Heller, A. Hacker, R. Gruellenberger, J. Innerhofer, H. Passath, C. Wagner, G. Haitchi, D. Flotzinger, R. Pacher, P. Wach, Non-invasive beat-to-beat cardiac output monitoring by an improved method of transthoracic bioimpedance measurement, Comp. Biol. Med. 36 (2006) 1185-1203.

[13] H.G. Hinghofer-Szalkay, N. Goswami, A. Rossler, E. Grasser, D. Schneditz, Reactive hyperemia in the human liver, Am. J. Physiol. Gastrointest. Liver Physiol. 295 (2008) G332-G337.
[14] M. Mlynarik, A. Makatsori, I. Dicko, H.G. Hinghofer-Szalkay, D. Jezova, Postural changes associated with public speech tests lead to mild and selective activation of stress hormone release, J. Physiol. Pharmacol. 58 (2007) 95-103.

[15] A. Dutta, S. Bhattacharya, L.H. Keel, Linear Control Theory: Structure, Robustness and Optimization, CRC press, Florida, 2009.

[16] W.R. Lovallo, Stress and Health: Biological and Psychological Interactions, Sage Publications, Thousand Oaks, 1997.

[17] J.R. Turner, D. Carroll, Heart rate and oxygen consumption during mental arithmetic, a video game, and graded exercise: further evidence of metabolically exaggerated cardiac adjustments?, Psychophysiol 22 (1985) 261-267. 